\title{
Nonundershooting linear multivariable tracking controllers ${ }^{\star}$
}

\author{
Robert Schmid* Lorenzo Ntogramatzidis ** \\ ${ }^{*}$ Department of Electrical and Electronic Engineering, University of \\ Melbourne, Parkville, VIC 3010, Australia. (E-mail: \\ rschmid@unimelb.edu.au) \\ ** Department of Mathematics and Statistics, Curtin University, Perth WA \\ 6845, Australia. (E-mail: L.Ntogramatzidis@curtin.edu.au).
}

\begin{abstract}
We consider the use of linear multivariable state feedback control to achieve a nonundershooting step response. The recently introduced nonovershooting linear state feedback controller design method of Schmid and Ntogramatzidis (2010) is here modified to yield a step response that is nonundershooting. The design method is applicable to square and non-square systems, minimum and nonminimum phase systems, and also strictly proper and bi-proper systems.
\end{abstract}

\section{INTRODUCTION}

The problem of designing a linear control law to achieve a nonundershooting or nonovershooting step response for linear time invariant (LTI) systems has been studied for several decades. It is well known that the transient response of an LTI system is related to its zero structure, and numerous studies have reported fundamental performance limitations arising from nonminimum phase (NMP) zeros. A recent comprehensive survey of the impact of system zeros on control system performance is given in Hoagg and Bernstein (2007).

Much of the existing literature on overshoot and undershoot is concerned with single-input single-output (SISO) systems. Papers offering analytic results on the system overshoot include Stewart and Davison (2006) where it was shown that for a continuous-time SISO system with two nonminimum phase real zeros (right-hand complex plane), the step response must overshoot if the settling time is sufficiently small. Analytic results on the system undershoot include Middleton (1991), where it is shown that an LTI SISO continuous-time system has an undershooting step response if it contains at least one real nonminimum phase zero. A lower bound for the size of the undershoot is also given, and this result is extended in Lau et al. (2003) where SISO systems with two real nonminimum phase zeros are considered and a lower bound for the minimum undershoot is given. Johansson (2002) considered multi-input multi-output (MIMO) systems subject to dynamic output feedback, and gave a lower bound on the system undershoot and interaction for systems with at least one real NMP zero.

Papers simultaneously considering both undershoot and overshoot have generally sought conditions to ensure the step response is monotonic. In Anderson et al. (1996), the authors give conditions for discrete-time and continuous-time SISO systems to have a nonnegative realization, implying a positive impulse response and hence a monotonic step response. Papers offering design methods include Darbha (2003), which gave conditions are given for the existence of a controller to achieve a sign invariant impulse response, and hence also a monotonic

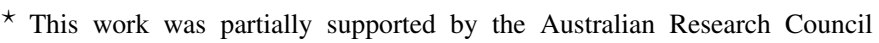
(Discovery Grant DP0986577).
}

step response. However such an approach is inherently conservative, because a monotonic step response is not necessary to avoid undershoot or overshoot.

To date there have been few papers offering analysis or design methods for overshoot or undershoot in the step response of MIMO systems. A recent contribution in this area is Schmid and Ntogramatzidis (2010), which gives design methods for a state feedback controller to yield a nonovershooting step response for LTI MIMO systems; the design method was applicable to some nonminimum phase systems, and could be applied to both continuous-time and discrete-time systems. In this paper we consider how these design methods may be modified to achieve a step response for MIMO systems that is nonundershooting. It should be noted that the question of whether a nonundershooting (or nonovershooting) tracking controller can be obtained for an arbitrary LTI system is an open question, even for SISO systems.

The paper is organized as follows. In Section 2 we formulate our tracking problem and formalize the definitions of undershooting step response. Section 3 briefly reviews the state feedback controller design method given in Schmid and Ntogramatzidis (2010), and explains how the eigenstructure assignment algorithm of Moore (1976) may be employed to obtain a closed-loop system in which only a small number of the system modes appear in each component of the output. Thus each component of the output may be rendered as the sum of just a few real exponentials. Section 4 then provides some conditions under which sums of real exponentials have real positive roots. In Section 5 these technical results are incorporated into the design method of Schmid and Ntogramatzidis (2010) to give a systematic procedure for obtaining a state feedback control law to yield a closed-loop system whose step response is nonundershooting.

In Section 6, the design method is applied to an example. We consider a MIMO system with three real nonminimum phase zeros, and obtain a step response that is nonundershooting. This example will show that the well-known result in Middleton (1991) according to which any SISO system with a real nonminimum phase zero must exhibit undershoot in its 
step response does not straightforwardly generalize to MIMO systems with real nonminimum phase zeros.

\section{PROBLEM FORMULATION}

Consider the LTI system $\Sigma$ governed by

$$
\Sigma:\left\{\begin{array}{l}
\dot{x}(t)=A x(t)+B u(t), \\
y(t)=C x(t)+D u(t),
\end{array}\right.
$$

where, for all $t \in \mathbb{R}, x(t) \in \mathbb{R}^{n}$ is the state, $u(t) \in \mathbb{R}^{m}$ is the control input, $y(t) \in \mathbb{R}^{p}$ is the output. For time $t<0$, we assume the system state is at an initial equilibrium $x_{0}$, the control input is at constant value $u_{0}$, and the output is at constant value $y_{0}=$ $C x_{0}+D u_{0}$. Here, $A, B, C$ and $D$ are appropriate dimensional constant matrices. We assume that $B$ has full column rank and $C$ has full row rank. We use $\mathbb{R}^{+}$to denote the set of positive real numbers, and $\mathbb{C}^{-}$to indicate the left hand complex plane. A complex number $\lambda \in \mathbb{C}$ is said to be stable if $\lambda \in \mathbb{C}^{-}$, and a stable matrix is one that has all its eigenvalues contained within $\mathbb{C}^{-}$. In this paper we are concerned with the problem of designing a linear state feedback control law for (1) such that the output $y$ of (1) tracks a step reference $r \in \mathbb{R}^{p}$ with zero steady-state error, and with zero undershoot and overshoot. We adopt the following standard assumption throughout this paper: Assumption 2.1. System $\Sigma$ is right invertible, stabilizable and has no invariant zeros at the origin.

This assumption ensures that a linear tracking controller can be obtained to track any reference target $r$ from any given initial condition $x_{0}$ as follows: choose a feedback gain matrix $F$ such that $A+B F$ is stable. Two vectors $x_{\mathrm{ss}} \in \mathbb{R}^{n}$ and $u_{\mathrm{ss}} \in \mathbb{R}^{m}$ exist that satisfy

$$
\begin{aligned}
& 0=A x_{\mathrm{ss}}+B u_{\mathrm{ss}} \\
& r=C x_{\mathrm{ss}}+D u_{\mathrm{ss}}
\end{aligned}
$$

for any $r \in \mathbb{R}^{p}$. Application of the control input

$$
u(t)=\left\{\begin{array}{cc}
u_{0}, & t<0 \\
F\left(x(t)-x_{\mathrm{ss}}\right)+u_{\mathrm{ss}}, & t \geq 0
\end{array}\right.
$$

yields an output

$$
y(t)=\left\{\begin{array}{cc}
y_{0}, & t<0 \\
C x(t)+D u(t), & t \geq 0
\end{array}\right.
$$

Employing the change of variable $\xi:=x-x_{\mathrm{ss}}$, we obtain the closed loop homogeneous system

$$
\Sigma_{\text {hom }}:\left\{\begin{array}{l}
\dot{\xi}(t)=(A+B F) \xi(t), \\
y(t)=(C+D F) \xi(t)+r .
\end{array}\right.
$$

Since $A+B F$ is stable, $x$ converges to $x_{\mathrm{ss}}, u$ converges to $u_{\mathrm{ss}}$, and $y$ converges to $r$ as $t$ goes to infinity. For a SISO system, overshoot means that the output exceeds the target $r \in \mathbb{R}$, while undershoot means that the output moves further away from the target than its initial distance. Since overshoot was the subject of the paper Schmid and Ntogramatzidis (2010), we will in this paper be concerned with the system undershoot. If $y_{0}<r$, undershoot occurs if the response $y(t)$ takes values less than or equal to $y_{0}$. If $y_{0}>r$, undershoot occurs if the response $y(t)$ takes values greater than or equal to $y_{0}$.

It is also helpful to understand the system response in terms of the tracking error $\varepsilon(t)=r-y(t)$. At the initial equilibrium $\left(x_{0}, u_{0}, y_{0}\right)$, we have $\varepsilon_{0}=r-y_{0}$. Thus for $y_{0}<r$, undershoot corresponds to the tracking error taking values greater than or equal to its initial value $\varepsilon_{0}$, while for $y_{0}>r$ it corresponds to the error taking values smaller than its initial value $\varepsilon_{0}$. Figure 1 shows some examples of step responses $y(t)$ and the corresponding tracking errors $\varepsilon(t)=r-y(t)$ with $y_{0}<r$. Notice that $y_{1}, y_{2}$ and $y_{3}$ are all nonundershooting step responses, while $y_{4}$ and $y_{5}$ do undershoot.
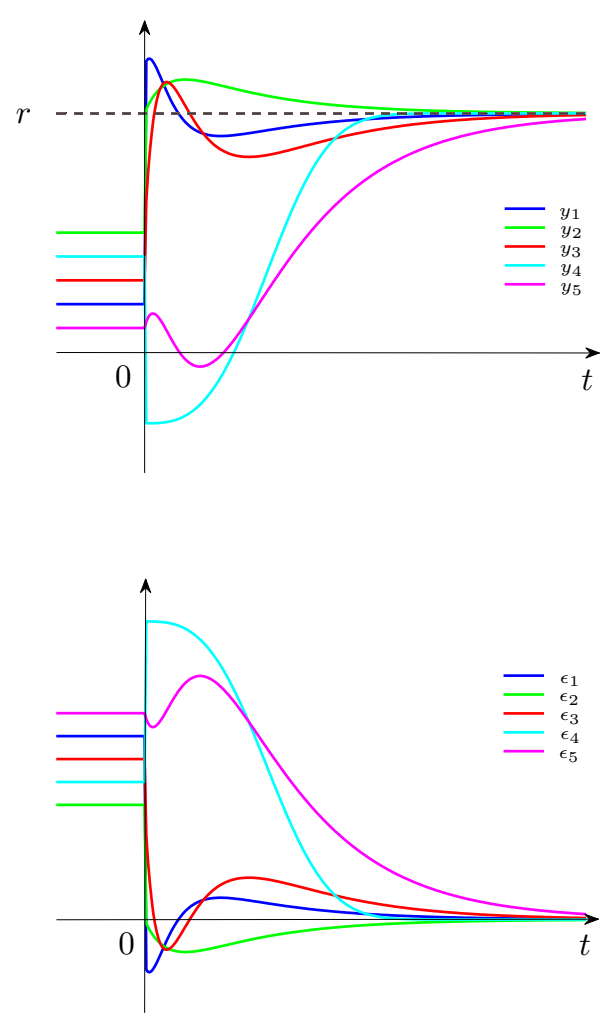

Fig. 1. Possible step response curves and corresponding tracking errors with $y_{0}<r$.

Note that for bi-proper systems (i.e., with $D \neq 0$ ), the instantaneous change in the control input at $t=0$ may lead to instantaneous changes in the output at $t=0$. Thus in general for $D \neq 0$, we have $y_{0} \neq y(0)$. For example, in Figure 1 we can see that the responses $y_{1}, y_{2}$ and $y_{4}$ are all characterized by an instantaneous transition from $y_{0}$ to $y(0)$ at $t=0$. For $y_{0}<r$, we must have $y_{0} \leq y(0)$, else undershoot occurs immediately. This is the case of $y_{4}$ in Figure 1.

To diagnose cases where overshoot and undershoot may occur instantaneously due to discontinuities in the output arising from $D \neq 0$, we may write the error $\varepsilon(0)=\mu \varepsilon_{0}$, for some $\mu \in \mathbb{R}$. Then $\mu>1$ corresponds to instantaneous undershoot, since the inequalities $\varepsilon(0)>\varepsilon_{0}$ and $\varepsilon(0)<\varepsilon_{0}$ in the cases $y_{0}<r$ and $y_{0}>r$, respectively, can be written as $\varepsilon(0)=\mu \varepsilon_{0}$ with $\mu>1$. For strictly proper systems we will always have $\mu=1$.

We now formalize the above discussion with the following definition.

Definition 2.1. Assume the initial state, control input and output of the system $\Sigma$ are given by $\left(x_{0}, u_{0}, y_{0}\right)$. Let $r \in \mathbb{R}^{p}$ be a step reference and let $\varepsilon=r-y$ be the tracking error obtained when the control input $u$ given in (4) is applied to $\Sigma$. Define $\varepsilon_{0}=r-y_{0}$, and for each component $k \in\{1, \ldots, p\}$, let $\varepsilon_{0, k}$ denote the $k$-th component of $\varepsilon_{0}$. Then 
(i) the system $\Sigma$ has an undershooting response in the output component $y_{k}(t)(k \in\{1, \ldots, p\})$ for a given $r \in \mathbb{R}^{p}$ from the initial condition $\left(x_{0}, u_{0}, y_{0}\right)$ if either (a) $\varepsilon_{k}(0)=\mu_{k} \varepsilon_{0, k}$, for some $\mu_{k}>1$, or (b) there is a $\bar{t} \in \mathbb{R}^{+}$such that $\varepsilon_{k}(\bar{t})=\varepsilon_{0, k}$;

(ii) if $\Sigma$ is nonundershooting in all output components for a given $r \in \mathbb{R}^{p}$, then we say $\Sigma$ has a nonundershooting response for the specified $\left(x_{0}, u_{0}, y_{0}\right)$ and $r$.

(iii) if $\Sigma$ is nonundershooting in all output components for a given $r \in \mathbb{R}^{p}$ from all initial conditions $\left(x_{0}, u_{0}, y_{0}\right)$, then we say $\Sigma$ has a globally nonundershooting response for this $r$.

Our aim in this paper is to choose the gain matrix $F$ such that $\Sigma$ has a nonundershooting response, for any given $r$ and $x_{0}$.

\section{FEEDBACK CONTROLLER DESIGN METHOD}

In Schmid and Ntogramatzidis (2010) a linear state feedback design method was introduced to yield a nonovershooting step response. The main idea of the method was to employ the classic result on eigenstructure assignment given by B.C. Moore in Moore (1976) to constrain the output $y(t)$ in such a way that the each component of the error term $\varepsilon(t)$ contained only a small number of the closed loop modes (poles). Here we summarize the design method, and present some results that describe the form of the error term. In Section 5 we consider how the design method may be employed to yield a closed loop response that is nonundershooting. A key result is the following eigenstructure lemma from Schmid and Ntogramatzidis (2010), which is itself an adaptation of Moore's algorithm.

Lemma 3.1. Schmid and Ntogramatzidis (2010) Let $\mathscr{L}=$ $\left\{\lambda_{1}, \ldots, \lambda_{n}\right\}$ be a self-conjugate set of $n$ distinct complex numbers. Let $\mathscr{S}=\left\{s_{1}, \ldots, s_{n}\right\}$ be a set of $n$ (not necessarily distinct) vectors in $\mathbb{R}^{p}$. Assume that, for each $i \in\{1, \ldots, n\}$, the matrix equation

$$
\left[\begin{array}{cc}
A-\lambda_{i} I & B \\
C & D
\end{array}\right]\left[\begin{array}{c}
v_{i} \\
w_{i}
\end{array}\right]=\left[\begin{array}{c}
0 \\
s_{i}
\end{array}\right]
$$

has solutions sets $\mathscr{V}=\left\{v_{1}, \ldots, v_{n}\right\} \subset \mathbb{C}^{n}$ and $\mathscr{W}=\left\{w_{1}, \ldots, w_{n}\right\} \subset$ $\mathbb{C}^{p}$. Then, provided $\mathscr{V}$ is linearly independent, a unique real feedback matrix $F$ exists such that, for all $i \in\{1, \ldots, n\}$,

$$
\begin{aligned}
& (A+B F) v_{i}=\lambda_{i} v_{i}, \\
& (C+D F) v_{i}=s_{i} .
\end{aligned}
$$

Proposition 1 of Moore (1976) shows explicitly how to construct $F$; we note that Moore's algorithm may readily be executed with MATLAB ${ }^{\circledR}$.

\subsection{Systems with $n-p$ minimum phase zeros}

The first design method applies to systems that satisfy

Assumption 3.1. System $\Sigma$ is square $(m=p)$ has at least $n-p$ distinct invariant zeros in $\mathbb{C}^{-}$.

Let $\mathscr{L}=\left\{\lambda_{1}, \ldots, \lambda_{n}\right\} \subset \mathbb{C}^{-}$denote the set of distinct stable closed loop eigenvalues of $A+B F$ to be chosen. Let $\left\{z_{1}, z_{2}, \ldots, z_{n-p}\right\} \subset \mathbb{C}^{-}$be freely chosen from among the distinct minimum phase invariant zeros of $\Sigma$. Then, we choose $\lambda_{i}=z_{i}$ for $i \in\{1, \ldots, n-p\}$; these modes are stable as all $z_{i}$ lie in $\mathbb{C}^{-}$. For $i \in\{n-p+1, \ldots, n\}$, the $\lambda_{i}$ may be freely chosen to be any real distinct stable modes not coincident with invariant zeros of $\Sigma$. Let $\left\{e_{1}, \ldots, e_{p}\right\}$ be the canonical basis of $\mathbb{R}^{p}$, and let $\mathscr{S}=\left\{s_{1}, \ldots, s_{n}\right\} \subset \mathbb{R}^{p}$ be such that

$$
s_{i}=\left\{\begin{array}{cl}
0 & \text { for } i \in\{1, \ldots, n-p\} \\
e_{1} & \text { for } i=n-p+1 \\
\vdots & \\
e_{p} & \text { for } i=n .
\end{array}\right.
$$

After solving (7) for the vectors in $\mathscr{S}$, we obtain sets $\mathscr{V}=$ $\left\{v_{1}, \ldots, v_{n}\right\} \subset \mathbb{C}^{n}$ and $\mathscr{W}=\left\{w_{1}, \ldots, w_{n}\right\} \subset \mathbb{C}^{p}$. Provided $\mathscr{V}$ is linearly independent, by Lemma 3.1, Moore's algorithm yields $F$ such that $A+B F$ has eigenstructure given by $\mathscr{L}$ and $\mathscr{V}$, respectively. The vectors in $\mathscr{V}$ satisfy

$$
\begin{aligned}
& (A+B F) v_{i}=\lambda_{i} v_{i}, \quad i \in\{1, \ldots, n\}, \\
& (C+D F) v_{i}= \begin{cases}0 & i \in\{1, \ldots, n-p\}, \\
e_{i-(n-p)} & i \in\{n-p+1, \ldots, n\} .\end{cases}
\end{aligned}
$$

Now introduce the new state coordinate $\xi:=x-x_{\text {ss }}$. As $\mathscr{V}$ is linearly independent, the matrix $V:=\left[\begin{array}{llll}v_{1} & v_{2} & \ldots & v_{n}\end{array}\right]$ is invertible. Introduce $\xi_{0}=x_{0}-x_{s s}$ and $\alpha:=\left[\begin{array}{llll}\alpha_{1} & \alpha_{2} & \ldots & \alpha_{n}\end{array}\right]^{\top}=V^{-1} \xi_{0}$. Theorem 3.1 describes the form of the error term:

Theorem 3.1. Schmid and Ntogramatzidis (2010). Assume $\Sigma$ satisfies Assumption 3.1. Let $\mathscr{L}$ be chosen as above, let $F$ be obtained from Moore's algorithm, let $r \in \mathbb{R}^{p}$ be any step reference, and let $x_{0} \in \mathbb{R}^{n}$ be any initial condition. Then, the error term $\varepsilon$ obtained from applying $u$ in (4) to $\Sigma$ has the form

$$
\varepsilon(t)=\left[\begin{array}{c}
\alpha_{n-p+1} e^{\lambda_{n-p+1} t} \\
\vdots \\
\alpha_{n} e^{\lambda_{n} t}
\end{array}\right] .
$$

We observe that each of the $p$ components of $\varepsilon$ contain exactly one mode.

The design method described in this section employs two rather simple ideas. Firstly, the available minimum phase invariant zeros are used to render the corresponding modes invisible in the tracking error via pole/zero cancelation. The remaining modes are distributed evenly into the $p$ components of the tracking error. Thus, in Theorem $3.1, n-p$ modes are canceled and the remaining $p$ modes are allocated with one mode per component.

\subsection{Systems with fewer than $n-p$ minimum phase zeros}

The design method given in Section 3.1 can be extended to systems with at least $n-l p$ minimum phase zeros, for any integer $l$, and by taking sufficiently large $l$ this includes all linear systems. The key idea is to channel $l$ modes per output component. Choose $\lambda_{i}=z_{i}$ for $i \in\{1, \ldots, n-l p\}$, and for $i \in\{n-l p+1, \ldots, n\}$ the $\lambda_{i}$ may be freely chosen to be any distinct real stable modes. Next let $\mathscr{S}=\left\{s_{1}, \ldots, s_{n}\right\} \subset \mathbb{R}^{p}$ be such that

$$
s_{i}=\left\{\begin{array}{l}
0 \text { for } i \in\{1, \ldots, n-l p\} \\
e_{1} \text { for } i \in\{n-l p+1, \ldots, n-l(p-1)\} \\
e_{2} \text { for } i \in\{n-l(p-1)+1, \ldots, n-l(p-2)\} \\
\vdots \\
e_{p} \text { for } i \in\{n-p+1, \ldots, n\} .
\end{array}\right.
$$

Solving (7) for the vectors in this new set $\mathscr{S}$, we obtain $\mathscr{V}=$ $\left\{v_{1}, \ldots, v_{n}\right\} \subset \mathbb{C}^{n}$ and $\mathscr{W}=\left\{w_{1}, \ldots, w_{n}\right\} \subset \mathbb{C}^{p}$, and provided $\mathscr{V}$ is linearly independent, Moore's algorithm yields $F$ such that $A+B F$ has distinct eigenvalues and eigenvectors given by $\mathscr{L}$ and $\mathscr{V}$, respectively. The following notation allows us to 
succinctly state our theorem for the form of the tracking error term in this case.

Notation 3.1. For each $k \in\{1, \ldots, p\}$, we let

(i) $v_{k, 1}, v_{k, 2}, \ldots, v_{k, l}$ denote the eigenvectors in $\mathscr{V}$ associated with canonical basis vector $e_{k}$ in (14), and let $\lambda_{k, 1}, \lambda_{k, 2}, \ldots \lambda_{k, l}$ be the corresponding eigenvalues in $\mathscr{L}$, ordered such that $\lambda_{k, 1}<\lambda_{k, 2}<\cdots<\lambda_{k, l}$ in each case;

(ii) For any $x \in \mathbb{R}^{n}$, introduce the new state coordinates $\xi=x-$ $x_{\mathrm{ss}}$ and let $\xi_{0}=\xi(0)$. Let $V$ be the matrix formed by the columns of $\mathscr{V}$, and let $\alpha:=V^{-1} \xi_{0}$ be the coordinate vector of $\xi_{0}$ in terms of $\mathscr{V}$. Then define

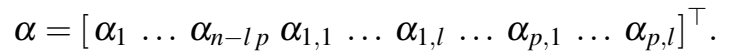

Theorem 3.2. Schmid and Ntogramatzidis (2010) . Assume $\Sigma$ has $n-l p$ minimum phase zeros. Let $\mathscr{L}$ be chosen as above, let $F$ be obtained from Moore's algorithm, let $r \in \mathbb{R}^{p}$ be any step reference, and let $x_{0} \in \mathbb{R}^{n}$ be any initial condition. Then $\varepsilon_{k}$, the $k$-th component of the error term $\varepsilon$ obtained from applying $u$ in (4) to $\Sigma$ has the form

$$
\varepsilon_{k}(t)=\alpha_{k, 1} e^{\lambda_{k, 1} t}+\alpha_{k, 2} e^{\lambda_{k, 2} t}+\cdots+\alpha_{k, l} e^{\lambda_{k, l} t} .
$$

Remark 3.1. We see that this eigenstructure based method for designing the feedback matrix $F$ gives us an error term that has a rather simple form; each component contains only $l$ distinct exponential terms. Other MIMO pole placement methods, for example those in Kautsky et al. (1985), that do not allow the designer to simultaneously select the eigenvectors lead to an error term in which all $n$ modes contribute in all $p$ output components. In Section 5 we introduce methods of exploiting the simplified form of the error term to obtain a suitable gain matrix $F$ that yields a nonundershooting step response.

\section{CONDITIONS IMPLYING UNDERSHOOT}

In this section we consider functions composed of sums of real exponential functions, and obtain some technical conditions under which these functions contain a real positive root. When applied to the system tracking error $\varepsilon$, such roots correspond to undershoot. The following result from the classic paper Laguerre (1883) provides information on the number of real roots of such functions in terms of the variations of the signs of their coefficients.

Notation 4.1. Let $l \in \mathbb{N}$, let $\left\{\lambda_{1}, \ldots, \lambda_{l}\right\}$ and $\left\{\alpha_{1}, \ldots, \alpha_{l}\right\}$ be sets of real numbers with $\lambda_{1}<\lambda_{2}<\cdots<\lambda_{l}$. Define $f: \mathbb{R} \rightarrow \mathbb{R}$ as

$$
f(t)=\alpha_{1} e^{\lambda_{1} t}+\alpha_{2} e^{\lambda_{2} t}+\cdots+\alpha_{l} e^{\lambda_{l} t} .
$$

We let $C\left\{\alpha_{1}, \ldots, \alpha_{l}\right\}$ denote the number of changes in the signs in the sequence of coefficients $\left\{\alpha_{1}, \alpha_{2}, \ldots, \alpha_{l}\right\}$, and for any interval $\mathscr{I} \subseteq \mathbb{R}, Z_{\mathscr{I}}^{f}$ denotes the number of real roots of $f$ in $\mathscr{I}$

Let us also introduce $p_{1}=\alpha_{1}, p_{2}=\alpha_{1}+\alpha_{2}, p_{3}=\alpha_{1}+\alpha_{2}+\alpha_{3}$, $\ldots, p_{l}=\alpha_{1}+\cdots+\alpha_{l}$ and $q_{1}=p_{1}\left(\lambda_{1}-\lambda_{2}\right), q_{2}=q_{1}+p_{2}\left(\lambda_{2}-\right.$ $\left.\lambda_{3}\right), \ldots, q_{l-1}=q_{l-2}+p_{l-1}\left(\lambda_{l-1}-\lambda_{l}\right), q_{l}=p_{l}$. Also introduce $r_{1}=\alpha_{l}, r_{2}=\alpha_{l}+\alpha_{l-1}, r_{3}=\alpha_{l}+\alpha_{l-1}+\alpha_{l-2}, \ldots, r_{l}=\alpha_{l}+$ $\cdots+\alpha_{1}$ and $s_{1}=r_{1}\left(\lambda_{l}-\lambda_{l-1}\right), s_{2}=s_{1}+r_{2}\left(\lambda_{l-1}-\lambda_{l-2}\right), \ldots$, $s_{l-1}=s_{l-2}+r_{l-1}\left(\lambda_{2}-\lambda_{1}\right), s_{l}=r_{l}$.

Lemma 4.1. (Laguerre, 1883, Section 24). Let $f$ be defined as in (17). Then

$$
\begin{aligned}
& Z_{(0,1)}^{f} \leq C\left\{q_{1}, \ldots, q_{l}\right\}, \\
& Z_{[1, \infty)}^{f} \leq C\left\{s_{1}, \ldots, s_{l}\right\} .
\end{aligned}
$$

Our first lemma gives a necessary and sufficient condition for the sum of two real exponentials to take on a specified non-zero value, which corresponds to undershoot.

Lemma 4.2. Let $\lambda_{1}<\lambda_{2}<0$, and for any real nonzero constants $\left\{\alpha_{1}, \alpha_{2}\right\}$, define

$$
f(t)=\alpha_{1} e^{\lambda_{1} t}+\alpha_{2} e^{\lambda_{2} t} .
$$

Let $\beta=\frac{1}{\mu}\left(\alpha_{1}+\alpha_{2}\right)$ for some $\mu \leq 1$. Then there exists $\bar{t} \in \mathbb{R}^{+}$ such that $f(\bar{t})=\beta$ if and only if $t^{*} \in \mathbb{R}^{+}$and $f\left(t^{*}\right) \beta \geq \beta^{2}$, where

$$
t^{*}=\frac{1}{\lambda_{2}-\lambda_{1}} \ln \left(\frac{-\lambda_{1} \alpha_{1}}{\lambda_{2} \alpha_{2}}\right) \text {. }
$$

Proof: Since $\lambda_{1}<\lambda_{2}<0$, function $f(t)$ goes to 0 as $t \rightarrow \infty$. Since $\beta=f(0) / \mu$, in the case $f(0)=\alpha_{1}+\alpha_{2}>0$, a $\bar{t}$ exists for which $f(\bar{t})=\beta$ if and only if $f(t)$ has a maximum whose value exceeds $\beta$, i.e., there exists $t^{*}>0$ such that $\dot{f}\left(t^{*}\right)=0$ and $f\left(t^{*}\right) \geq \beta$. If $f(0)<0$, such $\bar{t}$ exists if and only if $f(t)$ has a minimum whose value is more negative than $\beta$, i.e., a $t^{*}>0$ exists such that $\dot{f}\left(t^{*}\right)=0$ and $f\left(t^{*}\right) \leq \beta$. Setting $\dot{f}\left(t^{*}\right)=0$ yields $t^{*}$ given by (19). If $t^{*} \notin \mathbb{R}^{+}$, then $f$ is monotonic on $\mathbb{R}^{+}$ and takes its extreme value at $t=0$, implying $|f(t)|<\mid \alpha_{1}+$ $\alpha_{2}|\leq| \beta \mid$ for all $t \in \mathbb{R}^{+}$. If $t^{*} \in \mathbb{R}^{+}$, then $f$ takes its extreme value at $t=t^{*}$. As such, $\bar{t} \in \mathbb{R}^{+}$satisfying $f(\bar{t})=\beta$ exists if and only $f\left(t^{*}\right) \beta \geq \beta^{2}$, which captures both the condition $f\left(t^{*}\right) \geq \beta$ when $f(0)>0$ and the condition $f\left(t^{*}\right) \leq \beta$ when $f(0)<0$.

When we apply Lemma 4.2 to test for undershoot, each $\varepsilon_{k}(t)$ has the form (18), and we use $\beta_{k}=\varepsilon_{0, k}$ for the initial output. Also $\varepsilon_{k}(0)=\alpha_{1}+\alpha_{2}$, and we find $\mu_{k} \in \mathbb{R}$ such that $\mu_{k} \varepsilon_{0, k}=$ $\varepsilon_{k}(0)$. We only need consider $\mu \leq 1$ in Lemma 4 .2 because $\mu>$ 1 implies instantaneous undershoot. The following corollary offers a simpler result that can be used for strictly proper systems where no initial discontinuity in the output can occur.

Corollary 4.1. Under the assumptions of Lemma 4.2, if $\mu=1$, then there exists $\bar{t} \in \mathbb{R}^{+}$such that $f(\bar{t})=\beta$ if and only if and $\left(\alpha_{1}+\alpha_{2}\right)\left(\lambda_{1} \alpha_{1}+\lambda_{2} \alpha_{2}\right)>0$.

Proof: Here $\bar{t} \in \mathbb{R}^{+}$satisfying $f(\bar{t})=\beta$ exists if and only if $f(0) \dot{f}(0)>0$. This holds if and only $\left(\alpha_{1}+\alpha_{2}\right)\left(\lambda_{1} \alpha_{1}+\lambda_{2} \alpha_{2}\right)>$ 0 .

The next lemma gives conditions on when the sum of three real exponentials takes on a specified non-zero value.

Lemma 4.3. Let $\lambda_{1}<\lambda_{2}<\lambda_{3}<0$, and for any nonzero constants $\left\{\alpha_{1}, \alpha_{2}, \alpha_{3}\right\}$, define $\beta=\frac{1}{\mu}\left(\alpha_{1}+\alpha_{2}+\alpha_{3}\right)$ for some $\mu \leq 1$. Let

$$
f(t)=\alpha_{1} e^{\lambda_{1} t}+\alpha_{2} e^{\lambda_{2} t}+\alpha_{3} e^{\lambda_{3} t}
$$

and introduce $p_{1}=\alpha_{1}, p_{2}=\alpha_{1}+\alpha_{2}, p_{3}=\alpha_{1}+\alpha_{2}+\alpha_{3}, p_{4}=$ $\alpha_{1}+\alpha_{2}+\alpha_{3}-\beta$ and $q_{1}=p_{1}\left(\lambda_{1}-\lambda_{2}\right), q_{2}=q_{1}+p_{2}\left(\lambda_{2}-\lambda_{3}\right)$, $q_{3}=q_{2}+p_{3}\left(\lambda_{3}\right), q_{4}=p_{4}$. Also introduce $r_{1}=-\beta, r_{2}=\alpha_{3}-$ $\beta, r_{3}=\alpha_{3}+\alpha_{2}-\beta, r_{4}=\alpha_{1}+\alpha_{2}+\alpha_{3}-\beta$ and $s_{1}=r_{1}\left(-\lambda_{3}\right)$, $s_{2}=s_{1}+r_{2}\left(\lambda_{3}-\lambda_{2}\right), s_{3}=s_{2}+r_{1}\left(\lambda_{2}-\lambda_{1}\right), s_{4}=r_{4}$. Then there exists $\bar{t} \in \mathbb{R}^{+}$such that $f(\bar{t})=\beta$ only if at least one of the following conditions hold:

I. $C\left\{q_{1}, q_{2}, q_{3}, q_{4}\right\} \geq 1$;

II. $C\left\{r_{1}, r_{2}, r_{3}, r_{4}\right\} \geq 1$.

Proof: Define $g(t)=f(t)-\beta$. Then $f(\bar{t})=\beta$ for some $\bar{t} \in \mathbb{R}^{+}$if and only $g(\bar{t})=0$. Then we may write $g$ in the form (17) with coefficients $\left\{\alpha_{1}, \alpha_{2}, \alpha_{3},-\beta\right\}$ and exponents 
$\left\{\lambda_{1}, \lambda_{2}, \lambda_{3}, 0\right\}$. Applying Lemma 4.1 to $g$, we see that $\bar{t}$ exists only if $C\left\{q_{1}, q_{2}, q_{3}, q_{4}\right\} \geq 1$ or $C\left\{r_{1}, r_{2}, r_{3}, r_{4}\right\} \geq 1$.

The generalisation of these lemmas to the sum of any finite number of real exponentials is given by the following:

Lemma 4.4. For some positive integer $l$, let $\lambda_{1}<\lambda_{2}<\cdots<$ $\lambda_{l}<0$, and for any non-zero constants $\left\{\alpha_{1}, \alpha_{2}, \ldots, \alpha_{l}\right\}$, define

$$
f(t)=\alpha_{1} e^{\lambda_{1} t}+\alpha_{2} e^{\lambda_{2} t}+\cdots+\alpha_{l} e^{\lambda_{l} t} .
$$

Let $\beta=\frac{1}{\mu}\left(\alpha_{1}+\cdots+\alpha_{l}\right)$ for some $\mu \leq 1$, and define $\alpha_{l+1}=-\beta$ and $\lambda_{l+1}=0$. Use the sets $\left\{\alpha_{i}: 1 \leq i \leq l+1\right\}$ and $\left\{\lambda_{i}: 1 \leq i \leq\right.$ $l+1\}$ to obtain sets $\left\{q_{i}: 1 \leq i \leq l+1\right\}$ and $\left\{s_{i}: 1 \leq i \leq l+1\right\}$ as in Lemma 4.1. Then there exists $\bar{t} \in \mathbb{R}^{+}$such that $\bar{f}(\bar{t})=\beta$ only if $C\left\{q_{i}: 1 \leq i \leq l+1\right\} \geq 1$ or $C\left\{s_{i}: 1 \leq i \leq l+1\right\} \geq 1$.

Proof: These follow straightforwardly from Lemma 4.1. In each case, if neither of the conditions hold, then the relevant function has no root in $\mathbb{R}^{+}$.

The conditions given here are necessary, and in some cases also sufficient, for the existence of $\bar{t} \in \mathbb{R}^{+}$that corresponds to undershoot. Hence if the appropriate conditions are not satisfied, undershoot does not occur. The conditions given depend only the coefficients $\left\{\alpha_{i}\right\}$ and the modes $\left\{\lambda_{i}\right\}$, and do not require simulating the system response.

\section{NONUNDERSHOOTING FEEDBACK CONTROLLERS}

In this section we see how the feedback design method described in Section 3 can be combined with the technical lemmas in Section 4 to give a systematic procedure for searching for a nonundershooting feedback controller. We assume an LTI system $\Sigma$ in the form (1), together with a specified initial condition $x_{0}$, initial control input $u_{0}$, and step reference $r$. The following algorithm seeks to obtain a suitable set of closed loop eigenvalues $\mathscr{L}$ and corresponding eigenvectors $\mathscr{V}$ to which the Moore algorithm may be applied to obtain the a state feedback gain matrix $F$ that yields a closed-loop nonundershooting step response.

\section{Algorithm 5.1.}

(1) Begin by determining the value of the integer $l$ to be used for the choice of targets in (14). Formally $l$ is defined as

$$
l=\left\lceil\frac{n-z}{p}\right\rceil,
$$

where $z$ is the number of minimum phase zeros of $\Sigma$ and for any real number $q,\lceil q\rceil$ denotes the next integer larger than $q$.

(2) For a given initial condition $x_{0}$ and reference $r$, determine $x_{s s}$ and $u_{s s}$ from (2)-(3) and hence also $\xi_{0}=x_{0}-x_{s s}$.

(3) Choose a desired interval $[a, b]$ of the real line (where $a<b<0$ ), and form a candidate set $\mathscr{L}$ of $n$ distinct closed-loop eigenvalues containing the $n-l p$ minimum phase zeros of $\Sigma$, and $p$ sets of $l$ eigenvalues chosen from within $[a, b]$.

(4) For the appropriate value of $l$, determine the target set $\mathscr{S}$ from (14). Then solve for the corresponding $\mathscr{V}$ and $\mathscr{W}$ in (7) and check if $\mathscr{V}$ is linearly independent. If it is not then return to Step 3 and choose an alternative set of eigenvalues within $[a, b]$.

(5) Obtain the coordinate vector $\alpha$ in (15), and hence obtain the components $\varepsilon_{k}$ of the tracking error $\varepsilon$ from (16), for each $k \in\{1, \ldots, p\}$.
(6) For strictly proper systems, proceed directly to Step 7. For bi-proper systems, solve $\varepsilon_{k}(0)=\mu_{k} \varepsilon_{0, k}$ for $\mu_{k} \in$ $\mathbb{R}$, for each $k \in\{1, \ldots, p\}$. For a step response without instantaneous undershoot, check $\mu_{k}<1$ for each $k \in$ $\{1, \ldots, p\}$. If not return to Step 3 .

(7) If $l=1$, proceed directly to Step 8 . For $l \geq 2$, do the following for each $k \in\{1, \ldots, p\}$ : For a nonundershooting response, test each $\varepsilon_{k}$ for the conditions in Lemma 4.2 (if $l=2$ ), Lemma 4.3 (if $l=3$ ) or Lemma 4.4(b) (if $l \geq 4$ ) respectively. In each case, if none of the conditions in the respective lemmas are satisfied for any $k \in\{1, \ldots, p\}$, then $\mathscr{L}$ and $\mathscr{V}$ are satisfactory. If not, then return to Step 3.

(8) Apply Moore's algorithm to this $\mathscr{L}, \mathscr{V}$ and $\mathscr{W}$ to obtain the feedback matrix $F$ for $u$ in (4).

According to Theorems 3.1 and 3.2, the components of the closed-loop tracking error vector $\varepsilon$ have the form of (13) or (16), respectively. The tests in Step 7 ensure the coefficients $\alpha_{k, l}$ are such that undershoot does not occur in any of the output components. For the case $l=1$, the response is globally nonundershooting.

If $\Sigma$ has $n-(l-1) p+q$ stable zeros, where $1 \leq q<p$, then by (22) we would need to use the integer $l$ for the choice of targets in (14), even though $\Sigma$ has more than $n-l p$ stable zeros. However the additional $q$ stable zeros can be exploited by modifying (14) slightly. The $n$ closed loop modes in $\mathscr{L}$ can be associated with targets $s_{i}$ as follows: choose $s_{i}=0$ for $i \in\{1, \ldots, n-(l-1) p+q\}$, to fully exploit the available stable zeros. Then the first $q$ canonical basis vectors $\left\{e_{1}, \ldots, e_{q}\right\}$ needs to be associated with only $l-1$ modes each. The remaining $p-q$ canonical basis vectors $\left\{e_{q+1}, \ldots, e_{p}\right\}$ must be associated with $l$ modes.

Thus Algorithm 5.1 searches for suitable $\mathscr{L}$ and $\mathscr{V}$, but there is no guarantee that they can always be found, for any given $\left(x_{0}, u_{0}, y_{0}\right)$ and $r$, even if $[a, b]=(-\infty, 0)$ is chosen. Nonetheless in practice the search algorithm provides an effective tool for obtaining a nonundershooting linear controller when they do exist, due to the simplicity of the output function. Also the mathematical tests by which candidate sets of closed loop poles may be tested for suitability are computationally very tractable within MATLAB ${ }^{\circledR}$, allowing for a large number of candidate sets of poles to be tested in an efficient manner. Very recently a public domain toolbox, known as NOUS, has been developed to implement Algorithm 5.1; see Pandey and Schmid (2011).

An interesting aspect of the search algorithm is that it is generally more effective for MIMO systems than for SISO systems, as increasing the number of outputs $p$ reduces the value of $l$, meaning that fewer modes need to be channeled into each component of the output. This increased effectiveness may be explained by recalling that we have assumed $\Sigma$ is square; hence increasing $p$ also means increasing $m$, and thus we have more control inputs with which to determine the eigenstructure and hence shape the transient response. Finally note that as Lemmas 4.3 and 4.4 only offer necessary conditions, so using them in Step 7 will reject some $\mathscr{L}$ that do in fact yield suitable $F$ for a nonundershooting response.

\section{EXAMPLES}

A well known classic result on the relation between nonminimum phase zeros and the transient response is the following: 
Theorem 6.1. Middleton (1991). Let $\Sigma$ be an LTI stable strictly proper SISO system with at least one real NMP zero. Then the step response must exhibit undershoot.

The following conjecture is a possible MIMO generalization of this result:

Conjecture 6.1. Let $\Sigma$ be an LTI stable strictly proper square MIMO system with $p$ inputs/outputs, and $p$ real NMP zeros. Then the step response must exhibit undershoot in at least one output component.

Clearly, the case $p=1$ is given by Theorem 6.1 . We show this conjecture is false for MIMO systems, in general.

Example 6.1. Consider the strictly proper system $\Sigma_{1}$ :

$A=\left[\begin{array}{lllllllllll}0 & -5 & 0 & 6 & 8 & 0 ; 0 & 0 & 0 & -2 & 0 & 0\end{array}\right.$

$6 \begin{array}{lllllllllll}6 & 0 & 0 & 0 & 0 & 0 ; 0 & 0 & 0 & 0 & 0 & 0 \text {; }\end{array}$

$\left.\begin{array}{llllllllllll}0 & -1 & 0 & -6 & 3 & 0 ; 0 & 0 & 0 & 6 & 0 & 9\end{array}\right]$

$\mathrm{B}=\left[\begin{array}{lllllllllllllllll}1 & 0 & 0 & ; & 7 & 0 & 9 ; & 0 & 4 & -5 ; 0 & 0 & 7 ; 0 & 2 & 10 ;-2 & -1 & 0\end{array}\right]$

$\mathrm{C}=\left[\begin{array}{llllllllllllllll}0 & 7 & 0 & -2 & -3 & 0 ; 0 & 0 & -9 & -1 & 0 & 0 ; 0 & 0 & 7 & 6 & 5 & 0\end{array}\right]$

$\mathrm{D}=\left[\begin{array}{cccccccc}0 & 0 & 0 ; 0 & 0 & 0 ; 0 & 0 & 0\end{array}\right]$

The system has $n=6$ states, $p=3$ inputs and outputs, and $p=3$ real NMP zeros, at $0.5551,2.4547$ and 9.0000. We assume zero initial conditions and a step reference of $[1,1,1]^{T}$. Using Algorithm 5.1, we obtain the gain matrix $F$ as

$$
\begin{aligned}
& F=\left[\begin{array}{lllllll}
24.1 & -38.0 & -20.9 & 27.5 & 2.1 & -107.7
\end{array}\right.
\end{aligned}
$$

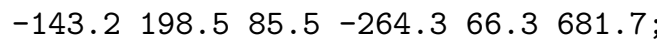

$$
\begin{aligned}
& \begin{array}{lllllll}
-88.0 & 111.9 & 45.9 & -179.7 & 51.0 & 378.1]
\end{array}
\end{aligned}
$$

so that the control law (4), with $u_{s s}$ and $x_{s s}$ obtained from solving (2)-(3), yields transient response curves as shown in Figure 2.
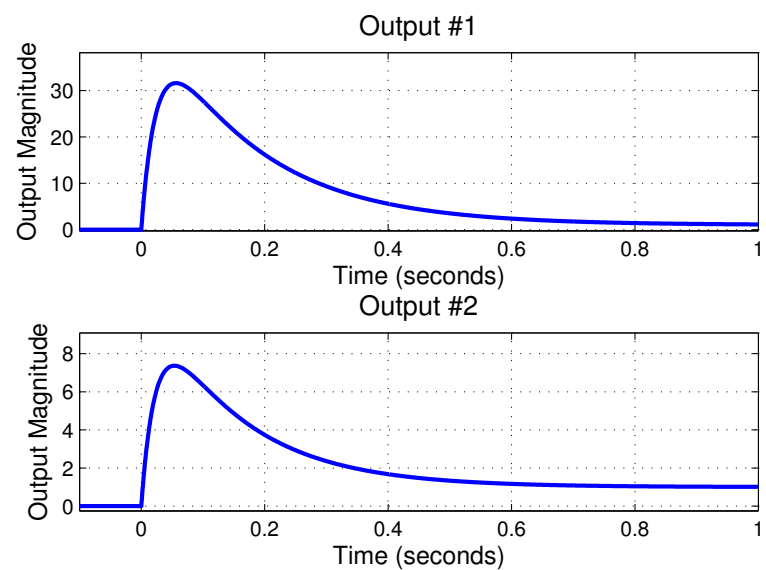

Output \#3

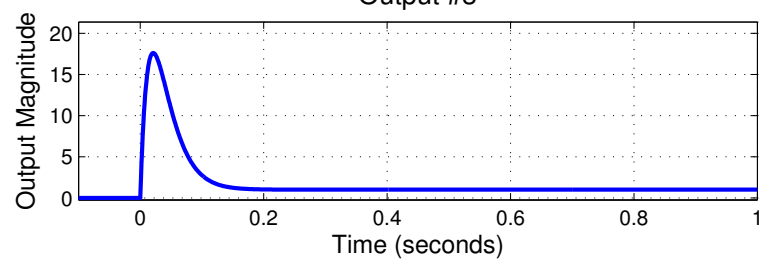

Fig. 2. Outputs from System $\Sigma_{1}$

The gain matrix $F$ places the closed-loop poles at

$\{-50,-47,-42,-40,-7,-6\}$, and of course the closedloop system has the same zeros as the open loop system, since state feedback has been used. Thus the closed-loop system is stable, strictly proper and square, with $p=3$ real NMP zeros. We see that with this control law, the step response does not exhibit undershoot in any of its outputs, indicating the conjecture is not valid in general. The authors have also obtained examples to disprove the conjecture for the cases $p=2, p=4$ and $p=5$.

\section{CONCLUSION}

The design method for a linear state feedback tracking controller to achieve a nonovershooting step response for MIMO systems given in Schmid and Ntogramatzidis (2010) has been modified here to yield a step response that is nonundershooting. The method is described here for continuous-time square systems, and is applicable to both strictly proper and bi-proper systems, which may be of minimum or nonminimum phase. The method may also be applied to discrete-time systems, and also non-square systems; we refer the reader to Schmid and Ntogramatzidis (2010) for the details. To the best of the authors' knowledge, this is the first linear control scheme that can provide a nonundershooting step response for a MIMO system.

\section{REFERENCES}

B.D.O. Anderson, M. Deistler, L. Farino and L. Benvenuti "Negative realization of a linear system with nonnegative impulse response", IEEE Transactions on Circuits and Systems-I: Fundamental Theory and applications, vol. 43(2), pp. 134-142, 1996.

S. Darbha, "On the synthesis of controllers for continuous time LTI systems that achieve a non-negative impulse response", Automatica, vol. 39, pp. 159-165, 2003.

J.B. Hoagg and D.S. Bernstein, "Nonminimum-Phase Zeros", IEEE Control Systems Magazine, pp. 45-57, 2007.

K.H. Johansson, "Interaction bounds in multivariable control systems", Automatica, Vol 38, pp. 1045-1051, 2002.

J. Kautsky, J. N.K. Nichols and P. Van Dooren, "Robust Pole Assignment in Linear State Feedback," International Journal of Control., vol. 41, pp. 1129-1155, 1985.

E. Laguerre, "On the Theory of Numeric Equations", Journal de Mathématiques pures et appliquées, $3^{e}$ série, t. IX, 1883. Translated by Stewart A. Levin, available from http://sepwww.stanford.edu/oldsep/stew/laguerre.pdf

K. Lau, R.H. Middleton and J.H. Braslavsky, "Undershoot and Settling Time Trade-offs for Nonminimum phase systems", IEEE Transactions on Automatic Control, vol. 48, pp. 13891393, 2003.

R.H. Middleton, "Trade-offs in linear control system design", Automatica, vol 27, 1991, pp. 281-292.

B.C. Moore, "On the Flexibility Offered by State Feedback in Multivariable systems Beyond Closed Loop Eigenvalue Assignment", IEEE Transactions on Automatic Control, vol. 21(5), pp. 689-692, 1976.

A. Pandey and R. Schmid, "NOUS: a MATLAB ${ }^{\circledR}$ toolbox for the design of nonovershooting and nonundershooting multivariable tracking controllers", available from http://people.eng.unimelb.edu.au/rschmid/documents/ NOUS1.0.zip

R. Schmid and L. Ntogramatzidis, "A unified method for the design of non-overshooting linear multivariable state-feedback tracking controllers", Automatica, pp. 312-321, 2010.

J. Stewart and D.E. Davison, "On Overshoot and Nonminimum phase Zeros" IEEE Transactions on Automatic Control, vol. 51(8), pp. 1378-1382, 2006. 